\title{
The ion-acoustic instability in the pre-flare plasma near the loop footpoints at solar active regions
}

\author{
A. Kryshtal ${ }^{1}$, S. Gerasimenko ${ }^{1}$, A. Voitsekhovska ${ }^{1}$, and V. Fedun ${ }^{2}$ \\ ${ }^{1}$ Department of Space Plasma, Main Astronomical Observatory of the National Academy of Sciences of Ukraine, \\ 27 Akademika Zabolotnoho St., 03680 Kyiv, Ukraine \\ ${ }^{2}$ Space Systems Laboratory, Department of Automatic Control and Systems Engineering, The University of Sheffield, \\ Mappin Street, Sheffield, S1 3JD, UK
}

Correspondence to: A. Kryshtal (annavoy@mao.kiev.ua)

Received: 25 March 2013 - Revised: 17 October 2013 - Accepted: 2 November 2013 - Published: 16 December 2013

\begin{abstract}
The necessary physical conditions for development of the ion-acoustic instability in the chromospheric part of a flaring loop current circuit are investigated. Two possible scenarios have been studied. First, we consider that preflare loop plasma with the large-scale sub-Dreicer electric field has a classical Coulomb conductivity and, second, when anomalous resistance appears due to saturation of Bernstein turbulence. The Fontenla-Avrett-Loeser (FAL) model of the solar atmosphere was used to describe the pre-flare plasma. We have shown that investigated instability can grow and develop either in the presence of the Coulomb conductivity or saturated Bernstein turbulence. We demonstrate that in the case of small-scale instability, the threshold value for the degree of nonisothermality is high and, therefore, cannot be reached by inclusion of the ordinary Joule heating. The ion-acoustic instability can develop at the pre-flare loop footpoints provided the electrons are more than 10 times hotter than the ions there.
\end{abstract}

Keywords. Solar physics, astrophysics, astronomy (photosphere and chromosphere)

\section{Introduction}

The problem of the solar atmosphere heating is directly linked to the studies of coronal magnetic loops (see e.g. Ionson, 1978; Duijveman et al., 1981; Holman, 1985; Fontenla et al., 2008; Gogoberidze et al., 2009, for details). Based on Vohkoh, SOHO and TRACE observational data, Aschwanden (2001) proposed two strategies for the further investigation of a heating problem: "(a) the inclusion of the chromosphere and transition region in conventional $\mathrm{AC}$ and DC models and (b) the exploration of magnetic reconnection models at lower heating efficiencies than in solar flares. Because coronal holes and the quiet Sun demand two orders of magnitude less heating than the coronal part that is topologically connected with active regions, the solution of the coronal heating problem has to be focused on the footpoint heated, filled, and over dense active region loops."

The presence of a wide range of plasma instabilities in the solar atmosphere has been reported by McClements (1989) (Langmuir wave instability), Fontenla (2005) (FarleyBuneman instability (FBI)), Voitenko and Goossens (2002) (kinetic Alfvén wave instability), etc. Some of these instabilities were studied in the context of the possible energy dissipation mechanisms (i.e. as processes) which are responsible for heating of the solar chromosphere. The influence of the Farley-Buneman instability on magnetohydrodynamic wave propagation has been analysed numerically by Fontenla (2005). Later, FBI was investigated further in order to obtain the revised semi-empirical models of the solar chromosphere, which vary from the quiet solar atmosphere Vernazza-Avrett-Loeser (VAL) and more recent FontenlaAvrett-Loeser (FAL) models (see Fontenla et al., 2008, 1993; Vernazza et al., 1981). The generation mechanism of smallscale density irregularities in the partially ionized plasma of the solar chromosphere by FBI was reported by Gogoberidze et al. (2009). These small-scale irregularities can be used for remote diagnostics of strong cross-field currents in the solar chromosphere. Observations confirm that energy release in the vicinity of the loop footpoint regions (i.e. at the 
chromospheric part of the loop's current circuit) is ubiquitous in the solar chromosphere (e.g. Melnikov et al., 2002).

In the present paper, we explore the excitation mechanisms of the ion-acoustic instability and necessary physical conditions for its development in the pre-flare plasma at the footpoint of a coronal flaring loop (see e.g. Kryshtal and Kucherenko, 1996), i.e. at the region of the loop's current circuit within the chromosphere and transition region. The FAL model of the solar atmosphere is taken into account (see Fontenla et al., 1993). We assume the presence of a strong (kilogauss) magnetic field $\boldsymbol{B}_{0}$ and large-scale, quasi-static, and weak electric field, i.e. sub-Dreicer field $\boldsymbol{E}_{0}$ (see e.g. Solanki, 1993; Miller et al., 1997). $\boldsymbol{B}_{0}$ and $\boldsymbol{E}_{0}$ are considered as stationary, uniform, parallel to each other and almost perpendicular to the solar photosphere surface.

The term "pre-flare" plasma is used in a very narrow sense. The stream (or "drift") electron velocity $\boldsymbol{u}$ with respect to the almost immovable ions is much less than the electron thermal velocity $\boldsymbol{V}_{\mathrm{Te}}$. For the time interval when the electron stream is effectively decelerated by the ions and, therefore, the percentage of "runaway" electrons is negligibly small, the stream velocity $\boldsymbol{u}$ can be expressed as

$\boldsymbol{u}=\frac{e \boldsymbol{E}_{0}}{m_{\mathrm{e}} v_{\mathrm{ei}}}$,

where $\boldsymbol{E}_{0}$ is the electric field, $m_{\mathrm{e}}$ and $e$ are the mass and electron charge, and $v_{\mathrm{ei}}$ is the frequency of electron-ion collisions. For pair Coulomb collisions, the frequency of electron-ion collisions is (see e.g. Alexandrov et al., 1988)

$\nu_{\mathrm{ei}}=2 \cdot 10^{-6} n \frac{\ln \Lambda}{T_{\mathrm{e}, \mathrm{eV}}^{3 / 2}}$

where $n$ is the plasma density, $\ln \Lambda$ is the "Coulomb $\log$ arithm" and $T_{\mathrm{e}, \mathrm{eV}}$ is the electron temperature expressed in electron volts. By taking into account the well-known formula for the amplitude of the local Dreicer field $E_{\mathrm{D}}$ (Alexandrov et al., 1988), the condition for pre-flare plasma can be described as

$\varepsilon_{\mathrm{R}} \equiv \frac{E_{0}}{E_{\mathrm{D}}}=\frac{u}{V_{\mathrm{Te}}} \ll 1$.

The additional Stark broadening for the Balmer $H_{\beta}$ lines with principle quantum numbers $N>8$ confirms the existence of large-scale quasi-static electric fields in the solar atmosphere (Foukal and Hinata, 1991). According to the flare model proposed by Heyvaerts et al. (1977), the preheating phase of the flare process precedes the impulsive (i.e. "flash" phase). During this phase, Buneman instability develops when stream velocity $u$ exceeds the electron thermal velocity $V_{\mathrm{Te}}$. In this sense, we studied the phase of the flare process that precedes the preheating phase, which only exists if sub-Dreicer field $E_{0}$ is present. From a physical and mathematical point of view, the ion-acoustic instability has interesting and unique properties. First, this instability can be investigated by pure analytical methods (i.e. without making physical assumptions from the stage of its excitation up to saturation of ionacoustic turbulence). Second, the growth of ion-acoustic turbulence leads to the fast heating of a large plasma volume. Third, under additional conditions the ion-acoustic turbulence can be an important element in the formation of the pre-flare current layers (Somov et al., 1987).

\section{Physical approximations and basic formulas}

In present work, the ion-acoustic instability of the preflare plasma has been studied near the loop footpoint (i.e. at heights $600<h<1400 \mathrm{~km}$ above the photosphere). In this region the pre-flare plasma can be considered as "dense" and "cool" with typical values for density and temperature (i.e. $3 \times 10^{10} \mathrm{~cm}^{-3} \leq n_{\mathrm{e}}=n_{\mathrm{i}} \leq 5 \times 10^{11} \mathrm{~cm}^{-3}$ and $5000 \mathrm{~K} \leq\left(T_{\mathrm{e}}\right)_{0}=\left(T_{\mathrm{i}}\right)_{0} \leq 8000 \mathrm{~K}$ respectively). For such a plasma, the condition of quasi-neutrality

$n_{\mathrm{e}}=n_{\mathrm{i}}=n$

is satisfied for the low-frequency waves (oscillations). Here $n_{\mathrm{e}}$ and $n_{\mathrm{i}}$ are the electron and ion number density. By taking into account that during the linear stage of the ionacoustic instability development the ratio $\Theta \equiv \frac{T_{\mathrm{e}}}{T_{\mathrm{i}}}$ can grow, for example, due to ordinary Joule heating, we assume that $\left(T_{\mathrm{e}}\right)_{0}=\left(T_{\mathrm{i}}\right)_{0}$ is satisfied for the initial electron $T_{\mathrm{e}}$ and ion $T_{\mathrm{i}}$ temperatures. It is reasonable to use $\Theta=1$ condition at the beginning of the development of ion-acoustic instability (Galeev and Sagdeev, 1973; Kryshtal and Gerasimenko, 2004), plasma heating (Duijveman et al., 1981), or at the initial phase of pre-flare current layer formation (Somov et al., 1987). But the most interesting problems begin after this stage. The main question is the following: how to satisfy $\frac{T_{\mathrm{e}}}{T_{\mathrm{i}}}=$ const $\gg 1$ condition for the values of constants more than 6. Thus this problem reduces to the problem of the fast heating of solar plasma, when electron temperature $T_{\mathrm{e}}$ grows rapidly with respect to the ion temperature $T_{\mathrm{i}}$. To explain this, it was proposed to take into account an additional "heat source". For example, it could be resonant absorption of Alfvénic surface waves (Ionson, 1978) or circular cross current in the vicinity of pre-flare current layer (Somov et al., 1987). Another way is to find an instability with threshold which is much less than the threshold of the ion-acoustic instability (see e.g. Galeev et al., 1972; Duijveman et al., 1981; Somov et al., 1987; Voitenko and Goossens, 2002; Kryshtal and Gerasimenko, 2004; Fontenla et al., 2008; Gogoberidze et al., 2009; Kryshtal et al., 2012, for details). Under some conditions these instabilities can transform into weak and then later into saturated turbulence. Thus anomalous resistivity can appear in plasma. Duijveman et al. (1981) have shown that classical resistivity (and classical Joule heating) cannot exceed $T_{\mathrm{e}} / T_{\mathrm{i}}>3.1$. The ion-acoustic instability plays the role of instability with an extremely low threshold. But 
even the ion-acoustic wave heating, which appears after the "stage of ion-cyclotron turbulence", cannot satisfy the condition $T_{\mathrm{e}} / T_{\mathrm{i}}>6.4$. Furthermore, Holman (1985) had shown that "inertial resistivity", which was proposed as the possible process to reach $T_{\mathrm{e}} / T_{\mathrm{i}}>10$ (see e.g. Duijveman et al., 1981), is negligible in comparison to the classical Coulomb resistivity. To the best of our knowledge, the problem of "additional heat sources" in the solar atmosphere (i.e. how to reach $\Theta>10$ ) is questionable.

In the present paper we have made an attempt to find the necessary conditions (in particular, the threshold values of $\Theta$ ) for ion-acoustic instability development, which is superimposed on the Bernstein wave turbulence. This means that considered instability cannot appear if $\Theta<\Theta_{\text {bound }}$. Here $\Theta_{\text {bound }}$ is the finite number, which can be vary for different types of turbulence. For a classical laboratory plasma, if the external electric $\boldsymbol{E}_{0}$ and magnetic $\boldsymbol{B}_{0}$ fields are absent, the inequality

$v_{\mathrm{ee}} \gg v_{\mathrm{ei}} \gg v_{\mathrm{ii}}$

is satisfied. If parallel electric and magnetic fields are taken into account, the inequality (Eq. 5) is violated and, therefore, a pure analytical solution is difficult to obtain. However, a phenomenological approximate solution can be found by assuming that the right-hand side of inequality (Eq. 5) remains practically unchanged and the left-hand side is not "strong":

$v_{\mathrm{ee}} \geq v_{\mathrm{ei}} \gg v_{\mathrm{ii}}$.

Let us further assume that

$v_{\mathrm{ei}} \gg v_{\mathrm{e} 0}, v_{\mathrm{i} 0}$

is satisfied for collision frequencies of electrons and ions with neutral atoms. By applying the Bhatnagar-Gross-Krook (BGK) model collision integral, the total contribution of all collisions can be expressed as (see e.g. Alexandrov et al., 1988)

$\sum_{\alpha, \beta} v_{\alpha, \beta}=\sigma v_{\mathrm{ei}},(\alpha, \beta=e, i)$,

where $\sigma$ is the free numerical parameter of the problem in the range of $1 \leq \sigma \leq \sigma_{\max }$. If $\sigma=1$, the development of the instability is most favourable and, therefore, ion-electron collisions dominate in the plasma. The opposite case (i.e. $\sigma=\sigma_{\max }$ ) corresponds to the situation when instability is completely suppressed by the Coulomb collisions. By neglecting the ion-electron collisions $v_{\mathrm{ei}}$, the condition of the weakness of electric field $\boldsymbol{E}_{0}$ can be expressed in the form (see e.g. Pines and Schrieffer, 1961)

$$
\frac{e\left|\boldsymbol{E}_{0}\right|}{k_{z} k_{\mathrm{B}} T_{\alpha}} \ll 1,(\alpha=e, i),
$$

where $k_{z}$ is the longitudinal (i.e. along the fields $\boldsymbol{E}_{0} \| \boldsymbol{B}_{0}$ ) component of the perturbation wave vector $|\boldsymbol{k}|=\sqrt{k_{z}^{2}+k_{\perp}^{2}}$ and $k_{\mathrm{B}}$ is Boltzmann's constant. The phase velocity of the ion-acoustic wave varies in the range

$V_{\mathrm{Ti}} \ll\left|\frac{\omega}{k_{z}}-u\right| \ll V_{\mathrm{Te}}$,

where $V_{\mathrm{Ti}}$ is the thermal velocity of singly charged (i.e. $Z=1$ ) ions. We assume that corrections, which appear due to have taken into account the Coulomb collisions, could be neglected in the standard dispersion relation for the ionacoustic waves:

$\omega_{\mathrm{r}}^{2}=\frac{k_{z}^{2} V_{\mathrm{s}}^{2}}{1+k_{\mathrm{L}}^{2}}$,

where $\omega_{\mathrm{r}}$ is the real part of frequency, $V_{\mathrm{s}}=\sqrt{\frac{\mathrm{k}_{\mathrm{B}} T_{\mathrm{e}}}{m_{\mathrm{i}}}}$ the ion sound velocity, $d_{\mathrm{e}}=\frac{V_{\mathrm{Te}}}{\omega \mathrm{pe}}$ the Debye radius and $\omega_{\mathrm{pe}}=\sqrt{\frac{4 \pi n e^{2}}{m_{\mathrm{e}}}}$ the electron plasma frequency. The standard plasma approximation is applicable when $k_{\mathrm{L}} \equiv k_{z} d_{\mathrm{e}} \leq 2 \pi$ (i.e. if the wavelength of the perturbation exceeds the Debye radius). By assuming that inequality $\sqrt{\frac{\beta_{\mathrm{i}}}{8}} \ll \frac{\left|k_{z}\right| \rho_{\mathrm{i}}}{\varepsilon_{\mathrm{R}} \beta_{\mathrm{A}}}$ is satisfied, the influence of curvature and torsion of the magnetic field lines can be neglected. Here $\beta_{\mathrm{A}}=\frac{V_{\mathrm{Te}}}{V_{\mathrm{A}}}, \beta_{\mathrm{i}}=\frac{8 \pi n k_{\mathrm{B}} T_{\mathrm{i}}}{B_{0}^{2}}$ is the ion plasma beta, $\rho_{\mathrm{i}} \equiv \frac{V_{\text {Ti }}}{\Omega_{\mathrm{i}}}$ is the ion cyclotron radius, $\Omega_{\mathrm{i}}$ is the ion cyclotron frequency and $V_{\mathrm{A}}$ is the Alfvén velocity. For ion-acoustic waves in the plasma with Coulomb conductivity, the amplitude of the external magnetic field $\boldsymbol{B}_{0}$ is not important. In the presence of anomalous resistivity, plasma waves can superimpose on turbulence. For example, in plasma with the Bernstein turbulence, the effective collision frequency of the electrons stream and pulsations of saturated Bernstein turbulence is higher than electron-ion collisions. This effective frequency $v_{\text {eff }}$ depends on the cyclotron frequency $\Omega_{\mathrm{e}} \equiv \frac{e B_{0}}{m_{\mathrm{e}} c}$ and, therefore, on the amplitude of magnetic field $\left|\boldsymbol{B}_{0}\right|$ (see e.g. Galeev and Sagdeev, 1973). The condition in Eq. (3) allows us to neglect the influence of runaway electrons. For very small values of $\varepsilon_{\mathrm{R}}$, the percentage of these electrons can be written as (see e.g. Alexandrov et al., 1988)

$\frac{N_{\mathrm{r}}}{N_{\mathrm{e}}} \cong \frac{1}{2 \pi} \exp \left(-\frac{1}{\varepsilon_{\mathrm{R}}}\right)$.

Here, $N_{\mathrm{r}}$ is the number of runaway electrons, and $N_{\mathrm{e}}$ is the total number of the electrons in the unit plasma volume. From Eq. (12) we obtain an estimate value for the upper limit of $\varepsilon_{\mathrm{R}} \ll 1$. It can be verified that, for $\varepsilon_{\mathrm{R}}>0.2$, the value $\frac{N_{\mathrm{r}}}{N_{\mathrm{e}}}$ becomes of the order of $\sim 10^{-3}$, and therefore we cannot neglect the influence of the runaway electron beams on the development of the ion-acoustic instability. Thus, we consider the value $\varepsilon_{\mathrm{R}}=0.15$ as the limit of applicability. The dispersion relation for the ion-acoustic waves in a semiconductor plasma with uniform external electric field has been obtained previously by Pines and Schrieffer (1961). By taking into account the contribution of Coulomb collisions, the dispersion 
relation for the same type of waves in the pre-flare plasma has been found by Kryshtal and Kucherenko (1996):

$D(\omega, \boldsymbol{k})=\operatorname{ReD}(\omega, \boldsymbol{k})+i \operatorname{ImD}(\omega, \boldsymbol{k})=0$,

where

$$
\begin{aligned}
& \operatorname{ReD}(\omega, \boldsymbol{k})=1+x_{\mathrm{e}}^{2}\left(1-y_{\mathrm{e}}^{2}\right)-\frac{x_{\mathrm{i}}^{2}}{y_{\mathrm{i}}^{2}}\left(1+\frac{3}{y_{\mathrm{i}}^{2}}\right), \\
& \operatorname{ImD}(\omega, \boldsymbol{k})=x_{\mathrm{e}}^{2}\left\{\sqrt{\frac{\pi}{2}} y_{\mathrm{e}} \exp \left(-\frac{y_{\mathrm{e}}^{2}}{2}\right)-\frac{e E_{0}}{k_{z} k_{\mathrm{B}} T_{\mathrm{e}}}\right\} \\
& +\frac{x_{\mathrm{i}}^{2}}{y_{\mathrm{i}}^{2}}\left\{\tilde{v}+\sqrt{\frac{\pi}{2}} y_{\mathrm{i}}^{3} \exp \left(-\frac{y_{\mathrm{i}}^{2}}{2}\right)+\frac{3 e E_{0}}{m_{\mathrm{i}} \omega^{2}}\right\}
\end{aligned}
$$

and

$$
\begin{aligned}
& x_{\alpha} \equiv \frac{\omega_{P \alpha}}{k_{z} V_{T \alpha}},(\alpha=e, i), y_{\alpha} \equiv \frac{\omega-k_{z} u_{\alpha}}{k_{z} V_{T \alpha}},(\alpha=e, i), \\
& u_{i} \approx 0 .
\end{aligned}
$$

The dispersion relation (Eq. 13) is obtained by assuming that the equilibrium ion velocity distribution function can be represented by the usual Maxwell distribution, and the equilibrium electron distribution function is a shifted Maxwell distribution with the shift velocity $\boldsymbol{u}$ (see Eq. 1). Two different expressions for $\tilde{v}$ have been used to investigate the development of the ion-acoustic instability: $\tilde{v}=\frac{\nu_{\text {ei }}}{\omega} \sigma$ and $\tilde{v}=\frac{8}{5} \frac{k_{z} V_{\mathrm{Ti}}^{2}}{\omega^{2}}+\frac{\nu_{\mathrm{ei}}}{\omega} \sigma$. The second one is uncertain due to the inclusion of the ion thermal motions. The instability growth rate can be calculated by using the following classical formula (see e.g. Alexandrov et al., 1988):

$$
\delta=-\left.\frac{\operatorname{ImD}(\omega, \boldsymbol{k})}{\frac{\partial}{\partial \omega} \operatorname{ReD}(\omega, \boldsymbol{k})}\right|_{\omega=\omega_{\mathrm{r}}},
$$

where $\omega_{\mathrm{r}}$ is described by Eq. (11). The reduced growth rate in the units of plasma frequency $\omega_{\mathrm{Pe}}$ can be represented as (see e.g. Kryshtal and Kucherenko, 1996)

$$
\frac{\delta}{\omega_{\mathrm{Pe}}}=\sqrt{\frac{\pi}{8}} \mu k_{\mathrm{L}} \frac{G_{\mathrm{E}}}{P_{\mathrm{E}}},
$$

where

$$
\begin{aligned}
& \mu \equiv \sqrt{\frac{m_{\mathrm{e}}}{m_{\mathrm{i}}}} \approx 0.0233,\left(\text { for }: m_{\mathrm{i}}=938.28 \mathrm{MeV}\right), \\
& G_{\mathrm{E}}=-\left(\frac{2 k_{*}}{\pi}\right)^{\frac{1}{2}} \frac{\left\{\varepsilon_{\mathrm{R}} \mu\left(2+6 k_{\mathrm{L}}^{2}+3 k_{\mathrm{L}}^{4}\right)+\sigma k_{*}^{\frac{3}{2}}\right\}}{\omega_{\mathrm{Pe}} k_{\mathrm{L}} \mu} v_{\mathrm{ei}} \\
& -\Theta^{\frac{3}{2}} \exp \left(-\frac{\Theta}{2 k_{*}}\right)+\left(\varepsilon_{\mathrm{R}} k_{*}^{\frac{1}{2}}-\mu\right) \exp \left\{-\frac{1}{2}\left(\mu k_{*}^{\frac{1}{2}}-\varepsilon_{\mathrm{R}}\right)^{2}\right\}, \\
& P_{\mathrm{E}}=k_{*}^{2}\left[1+6 k_{*} \Theta^{-1}\right]+\mu\left[\varepsilon_{\mathrm{R}} k_{*}^{\frac{1}{2}}-\mu\right],
\end{aligned}
$$

and $k_{*} \equiv 1+k_{\mathrm{L}}^{2}$. In solar/space plasmas, several types of plasma wave instabilities can develop simultaneously. Moreover, instabilities may exist simultaneously at different development stages. Instability with a higher excitation threshold may superimpose on the saturated turbulence (e.g. instability with lower excitation threshold). In the laboratory plasmas, the longitudinal instability (for example ion-acoustic or Langmuir wave instability) has a higher excitation threshold than quasi-perpendicular (with $\frac{k_{z}}{k_{\perp}} \ll 1$ ) instabilities (see e.g. Alexandrov et al., 1988; Galeev and Sagdeev, 1973). The instability of the second harmonic of oblique Bernstein modes in plasma with sub-Dreicer electric field and Coulomb conductivity has been investigated previously by Kryshtal (1998)and Kryshtal et al. (2012). Due to a small but non zero $k_{z}$ component of the wave vector $|\boldsymbol{k}| \equiv \sqrt{k_{z}^{2}+k_{\perp}^{2}}$, such an instability can be excited by the parallel streams of electrons in the contrast to the ordinary (i.e. non-oblique Bernstein wave) instability that is usually excited by the cross currents. The numerical values of the reduced growth rate $\Gamma$ of this instability are summarized in Table 1 . The highest threshold value for the amplitude of sub-Dreicer field $(\varepsilon)_{\text {bound }}$ is $1.10 \times 10^{-4}$, and the lowest obtained threshold value $\varepsilon_{\mathrm{R}}$ for the ion-acoustic instability is $4 \times 10^{-2}$.

Unfortunately, in the case of Bernstein turbulence, it is not possible to build a purely analytical solution for the effective frequency $v_{\text {eff }}$ that describes the transformation from the linear stage of the instability development to the saturated turbulence. To the best of our knowledge, the exact expression for $v_{\text {eff }}$ does not exist. Estimates for $v_{\text {eff }}$ have been found previously by Galeev et al. (1972) and Galeev and Sagdeev $(1973,1984)$. To model the situation in which the ion-acoustic instability is superimposed on the saturated Bernstein turbulence, the ion-electron collision frequency $v_{\mathrm{ei}}$ in Eq. (15) has to be replaced by the effective collision fre-

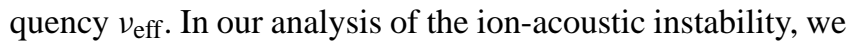
have applied three different expressions for $v_{\text {eff }}$ :

(a) $\frac{\nu_{\text {eff }}}{\Omega_{\mathrm{e}}} \sim \frac{\Gamma}{z_{\mathrm{e}}}$,

where $\Gamma$ is the reduced linear growth rate of the second harmonics of the Bernstein modes, $z_{\mathrm{e}} \equiv \frac{k_{\perp}^{2} V_{\mathrm{Te}}^{2}}{\Omega_{\mathrm{e}}^{2}} \equiv k_{\perp}^{2} \rho_{\mathrm{e}}^{2}$ (Galeev et al., 1972);

(b) $\frac{\nu_{\text {eff }}}{\Omega_{\mathrm{e}}}=\frac{\varepsilon_{\mathrm{R}}^{3}}{\ell}$,

where $\ell=10$ and $\ell=5$ for the first and second harmonics respectively (Galeev and Sagdeev, 1973, 1984);

(b) $\frac{v_{\text {eff }}}{\Omega_{\mathrm{e}}}=\frac{\varepsilon_{\mathrm{R}}^{3}}{25}\left[1+\left(\omega_{*} \varepsilon_{\mathrm{R}}^{-2}\right)\right]^{-2}$.

The limiting ratio of the longitudinal and transversal wavelengths of the Bernstein wave instability to the longitudinal 
Table 1. Values of principal characteristics of instability development of second oblique Bernstein harmonics.

\begin{tabular}{lrrrlr}
\hline $\begin{array}{l}\text { Models of the solar } \\
\text { atmosphere }\end{array}$ & $\left(\varepsilon_{\mathrm{R}}\right)_{\text {bound }} \times 10^{-4}$ & $\left(k_{\mathrm{L}}\right)_{\text {bound }}$ & $\left(z_{\mathrm{e}}\right)_{\text {bound }}$ & $\Gamma_{1(+)}$ & $h, \mathrm{~km}$ \\
\hline FAL P1 & 0.01 & 0.0156 & 0.02 & $1.49 \times 10^{-12}$ & 650 \\
FAL P2 & 0.95 & 0.0013 & 0.022 & $1.096 \times 10^{-6}$ & 700 \\
FAL P3 & 1.10 & 0.0012 & 0.021 & $1.41 \times 10^{-9}$ & 1380 \\
FAL P4 & 1.05 & 0.0174 & 0.019 & $2.76 \times 10^{-11}$ & 1375 \\
\hline
\end{tabular}

Table 2. Physical characteristics of the pre-flare plasma at the lower chromosphere of the loop's current circuit in an active region.

\begin{tabular}{llllllll}
\hline $\begin{array}{l}\text { Models of the solar } \\
\text { atmosphere }\end{array}$ & $n_{\mathrm{e}}, 10^{11} \mathrm{~cm}^{-3}$ & $\left(T_{\mathrm{e}}\right)_{0}=\left(T_{\mathrm{i}}\right)_{0}, \mathrm{~K}$ & $B_{0}, \mathrm{mT}$ & $h, \mathrm{~km}$ & $\omega_{\mathrm{Pe}}, 10^{10} \mathrm{~s}^{-1}$ & $\Omega_{\mathrm{e}}, 10^{10} \mathrm{~s}^{-1}$ & $v_{\mathrm{e}}, 10^{7} \mathrm{~s}^{-1}$ \\
\hline FAL P1 & 2.5 & 5220 & 321 & 650 & 2.81 & 5.64 & 2.56 \\
FAL P2 & 3.02 & 5480 & 321 & 700 & 3.10 & 5.64 & 2.89 \\
FAL P3 & 4.79 & 7420 & 145 & 1380 & 3.80 & 2.56 & 1.23 \\
FAL P4 & 0.31 & 6150 & 145 & 1375 & 0.97 & 2.56 & 0.093 \\
\hline
\end{tabular}

wavelengths of the ion-acoustic instability can be expressed as follows: $L_{\mathrm{R}} \equiv \frac{\left(\lambda_{z}\right)_{\mathrm{B}}}{\left(\lambda_{z}\right)_{\mathrm{IA}}}=\frac{\omega_{*}}{\sqrt{z_{\mathrm{e}}}} \frac{k_{\mathrm{L}}}{k_{\mathrm{R}}}, L_{\mathrm{R}}^{*} \equiv \frac{\left(\lambda_{\perp}\right)_{\mathrm{B}}}{\left(\lambda_{z}\right)_{\mathrm{IA}}}=\frac{\omega_{*}}{\sqrt{z}} k_{\mathrm{L}}$. Note that to avoid the possible resonant effects, the values of $L_{\mathrm{R}}$ and $L_{\mathrm{R}}^{*}$ should not be near unity. Formally, the saturated turbulence can decelerate instability as well as sustain it. Kryshtal et al. (2012) have shown that in the solar atmosphere described by the FAL model, the Bernstein wave instability has an extremely low threshold value. Therefore, the ion-acoustic instability and saturated Bernstein turbulence can develop simultaneously.

\section{Results and discussion}

In this work, we have analysed the ion-acoustic instability growth rate (see Eq. 15) for four modifications of the FAL model: FAL P1, FAL P2, FAL P3 and FAL A3 (see e.g. Fontenla et al., 1993). The ion-acoustic instability growth rate has been investigated in application to Machado-AvrettVernazza-Noyes (MAVN) (see e.g. Machado et al., 1980) and VAL (see e.g. Vernazza et al., 1981) models of the solar atmosphere previously by Kryshtal and Kucherenko (1996). Note that the differences between the the FAL and MAVN, and FAL and VAL models increase with height above the photosphere. The main physical characteristics of the preflare plasma at the footpoint of the loop (i.e. at $600<h<$ $1400 \mathrm{~km}$ ) are summarized in Table 2.

\subsection{The pre-flare plasma with the Coulomb conductivity}

In the case of pre-flare plasma with Coulomb conductivity, the ion-acoustic instability can develop only in the FAL P3 and FAL A3 modifications of the FAL model. In the FAL A3 model, the instability appears only at $\sigma=1$. Note that in plasma with the Coulomb conductivity, we have used the $v_{\mathrm{ei}}$ frequency in Eq. (15) for the growth rate of the ion-acoustic instability. The limit values for the ion-acoustic instability in the pre-flare plasma with Coulomb conductivity are summarized in Table 3.

The plot of the reduced growth rate $\Gamma$ as function of $\Theta$ and $k_{\mathrm{L}}$ for the FAL P3 model is shown in Fig. 1a. $\Gamma_{1(+)}$ describes the first positive value of the reduced growth rate (Eq. 15).

The positive growth rate corresponds to the right half space of the $\Gamma=\Gamma\left(\Theta, k_{\mathrm{L}}\right)$ with respect to the plane $\Gamma=0$. The existence of the curve $\Gamma=0$ confirms the possible evolution of the instability development regime (or wave damping) into the process of continuous generation of non-damped small amplitude ion-acoustic waves. Due to the small values of $\Gamma_{1(+)}$, the conversion through the region $\Gamma=0$ is smooth (i.e. without jumps and singularities). Note that these amplitudes are close to the thermal noise level. For the case of the FAL P3 model, we have found that the maximum value of the free numerical parameter $\sigma$ is equal to 4 . The ionacoustic instability appears to be small-scale: the limit values of the perturbation wavelengths vary between 20 and 60 Debye radii. Such values of Debye radii correspond to the $0.11 \leq\left(k_{\mathrm{L}}\right)_{\text {bound }} \leq 0.31$ range of the reduced wave vector limit (see Table 2). For waves with a high gain bandwidth and a growth rate that is much lower than the fundamental frequency, the linear stage of the development of the instability can continue for many wave periods. Therefore, such an instability cannot be aperiodic and can be classified as a wave process (Kadomtsev, 1988). The $\Theta_{\text {bound }}$ limit values are too high and, therefore, this is the weakness of the investigated instability. It is impossible to reach the lower limit of the $\Theta_{\text {bound }}=15$ by taking into account only Joule heating, and, therefore, additional energy sources required. 

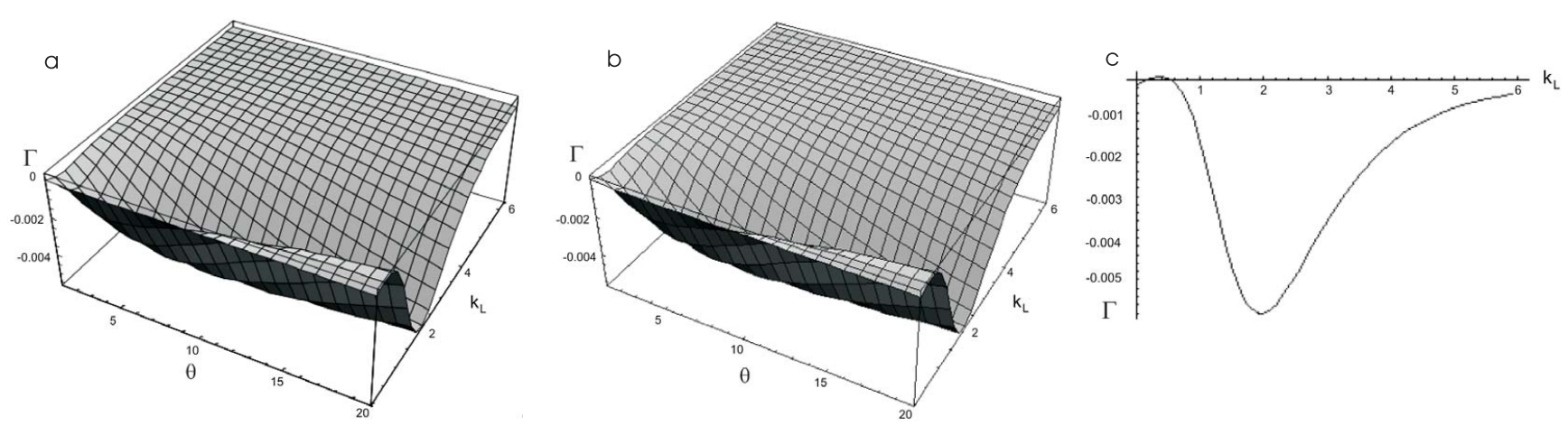

Fig. 1. (a) The reduced growth rate of the ion-acoustic instability in plasma with Coulomb conductivity for the FAL P3 model at $\left(\varepsilon_{\mathrm{R}}\right)_{\text {bound }}=$ $0.04, \sigma=1, \Theta_{\text {bound }}=17,\left(k_{\mathrm{L}}\right)_{\text {bound }}=0.11$ and $\Gamma_{1(+)}=1.33 \times 10^{-6} ;(\mathbf{b})$ the reduced growth rate of the ion-acoustic instability for the FAL P2 model at $\left(\varepsilon_{\mathrm{R}}\right)_{\text {bound }}=0.09, \Theta_{\text {bound }}=17,\left(k_{\mathrm{L}}\right)_{\text {bound }}=0.21$ and $\Gamma_{1(+)}=9.28 \times 10^{-6} ;(\mathbf{c})$ cross section of the surface $\Gamma=\Gamma\left(\Theta, k_{\mathrm{L}}\right)$ by the plane $\Theta=17$ for the FAL P2 model (see panel $\mathbf{b}$ ).

Table 3. Limit values of the ion-acoustic instability in the pre-flare plasma with Coulomb conductivity.

\begin{tabular}{lrrrrl}
\hline $\begin{array}{l}\text { Models of the solar } \\
\text { atmosphere }\end{array}$ & $\left(\varepsilon_{\mathrm{R}}\right)_{\text {bound }}$ & $\sigma$ & $(\Theta)_{\text {bound }}$ & $\left(k_{\mathrm{L}}\right)_{\text {bound }}$ & $\Gamma_{1(+)}$ \\
\hline FAL A3 & 0.099 & 1 & 17 & 0.21 & $8.2 \times 10^{-7}$ \\
FAL P3 & 0.099 & 1 & 15 & 0.11 & $1.25 \times 10^{-5}$ \\
FAL P3 & 0.1 & 2 & 15 & 0.21 & $7.3 \times 10^{-7}$ \\
FAL P3 & 0.1 & 3 & 17 & 0.21 & $1.44 \times 10^{-5}$ \\
FAL P3 & 0.102 & 4 & 17 & 0.31 & $3.7 \times 10^{-7}$ \\
\hline
\end{tabular}

\subsection{The pre-flare plasma with saturated Bernstein turbulence}

In the presence of anomalous resistivity, a small change in the reduced growth rate $\Gamma=\Gamma\left(\Theta, k_{\mathrm{L}}\right)$ can be observed for all three cases of the effective collision frequency $\nu_{\text {eff }}$ : Eqs. (16), (17) and (18). Firstly, in contrast to case (a), in the turbulent plasma the ion-acoustic instability can develop in the range $600<h<1400 \mathrm{~km}$ at all heights for four models: FAL P1, FAL P2, FAL P3 and FAL A3. Secondly, in the turbulent plasma the threshold value $\left(\varepsilon_{\mathrm{R}}\right)_{\text {bound }}$ can be more than two times less than for the plasma with Coulomb conductivity. This fact can result in a significantly higher energetic capacity of the pre-flare current layers (see e.g. Somov et al., 1987). Changes in the limit values of $\Theta$ and $k_{\mathrm{L}}$ are not important. A set of the limiting values demonstrates the minimal changes: $\Theta_{\text {bounds }}$ is equal to 13 instead of 15 in plasma with Coulomb conductivity. Therefore, the development of the ion-acoustic instability in plasma with saturated Bernstein turbulence is more preferable than in plasma with Coulomb collisions only. The range of $k_{\mathrm{L}}$ limit values remains practically the same (i.e. $0.11 \leq\left(k_{\mathrm{L}}\right)_{\text {bound }} \leq 0.31$ ). Also, the general tendency remains unchanged: the smaller value of $\left(\varepsilon_{R}\right)_{\text {bound }}$ corresponds to the higher value of $\Theta_{\text {bound }}$. Formally the set of $\Theta_{\text {bound }}$ values has no upper limit. In this study the set of $\Theta_{\text {bound }}$ values from 14 to 16 remains in the range $8.6 \leq \Theta \leq 16$, which has been obtained by Somov et al. (1987) for the thermal instability of a current sheet in the solar atmosphere. Note the thermal instability can lead to filamentation of current sheet. In plasma with $v_{\text {eff }}$ (Eq. 16), we used the limit values of $\Gamma_{1(+)}$ and $\left(z_{\mathrm{e}}\right)_{\text {bound }}$ for the Bernstein wave instability for the second harmonics of the oblique modes (see e.g. Kryshtal et al., 2012) to obtain the limit values of $\varepsilon_{\mathrm{R}}, \Theta$ and $k_{\mathrm{L}}$ for ion-acoustic instability. For the solar atmosphere models FAL P1, FAL P3 and FAL A3, the value of $\left(k_{\mathrm{L}}\right)_{\text {bound }}=0.11$ remains constant for any values of $\left(\varepsilon_{\mathrm{R}}\right)_{\text {bound }}$ and $(\Theta)_{\text {bound }}$. If $\left(\varepsilon_{\mathrm{R}}\right)_{\text {bound }}$ decreases from 0.1 up to 0.05 , the degree of nonisothermality $\Theta$ increases from 13 to 16 . For $\Theta_{\text {bound }} \leq 19$ the ion-acoustic instability in the case of FAL P2 has the values of normalized amplitude of the subDreicer field of $\left(\varepsilon_{\mathrm{R}}\right)_{\text {bound }} \in[0.06 ; 0.1]$, values of reduced longitudinal wave vector of $\left(k_{\mathrm{L}}\right)_{\text {bound }} \in[0.21 ; 0.31]$ and the values of degree of nonisothermality of $\Theta_{\text {bound }} \in[15 ; 19]$. In plasma with $v_{\text {eff }}$ (Eq. 17), the ion-acoustic instability develops for all four models.

In the case of FAL P1, FAL P3 and FAL A3, the values are $\left(k_{\mathrm{L}}\right)_{\text {bound }} \in[0.11 ; 0.31]$ and $(\Theta)_{\text {bound }} \in[15 ; 19]$, when $\left(\varepsilon_{\mathrm{R}}\right)_{\text {bound }}$ decreases from 0.1 to 0.04 . For the model FAL $\mathrm{P} 2$, the $\left(k_{\mathrm{L}}\right)_{\text {bound }}$ decreases from 0.21 to 0.11 and $\Theta_{\text {bound }}$ increases from 17 to 19 , when $\left(\varepsilon_{\mathrm{R}}\right)_{\text {bound }}$ decreases from 0.09 to 0.04 . 
The two-dimensional plot of $\Gamma=\Gamma\left(\Theta, k_{\mathrm{L}}\right)$, and the crosscut of $\Gamma=\Gamma\left(\Theta, k_{\mathrm{L}}\right)$ surface by plane $\left(\varepsilon_{\mathrm{R}}\right)_{\text {bound }}=0.09$ is shown in Fig. $2 b$ and c. This is a good example of the presence of local maximum of growth rate in the investigated region. In plasma with $v_{\text {eff }}$ (Eq. 18), the ion-acoustic instability exists for all four models. Note that $\left(k_{\mathrm{L}}\right)_{\text {bound }}=0.11$, when $\left(\varepsilon_{\mathrm{R}}\right)_{\text {bound }}$ decreases from 0.11 to 0.05 , and $\Theta_{\text {bound }}$ increases from 13 to 16. Finally, note that for FAL P2 and FAL P3 models, the values of the ratio of the wave length of the Bernstein instability to the wavelength of the ion-acoustic instability are $L_{\mathrm{R}} \simeq 282$ and $L_{\mathrm{R}} \simeq 360$, respectively. In the case of the FAL P1 and FAL A3 models, such values are $L_{\mathrm{R}} \simeq 67$ and $L_{\mathrm{R}} \simeq 14$, respectively. Therefore, for FAL P2 and FAL P3 we have $L_{\mathrm{R}}^{*} \simeq 0.28$ and $L_{\mathrm{R}}^{*} \simeq 0.36$, respectively. At the same time the $L_{\mathrm{R}}^{*} \simeq 0.07$ for FAL P1 and $L_{\mathrm{R}}^{*} \simeq 0.014$ for FAL A3. Thus, the last two models are more reasonable, due to satisfaction of the following inequalities: $L_{\mathrm{R}} \gg 1$ and $L_{\mathrm{R}}^{*} \ll 1$. These inequalities allow neglecting the resonant effects.

In this paper two possible scenarios - for plasma with Coulomb conductivity and for the plasma with the presence of saturated Bernstein turbulence - have been analysed in order to obtain the necessary conditions for development of the ion-acoustic instability at the footpoint region of preflare loops. It has been found that threshold values of degree of plasma nonisothermality remain too high for the usual Joule heating in both cases. Even after the appearance of saturated Bernstein wave turbulence, such values remain too high. This case needs further investigation. When condition $\Theta_{\text {bound }}>13$ is satisfied, we have shown that the inclusion of the anomalous resistivity facilitates development of ionacoustic instability, because it allows significant reduction of amplitude threshold value of the sub-Dreicer field. This is important for the formation of the pre-flare current layer and its energetic capability. In this case we have found the principal possibility of continuous generation of non-damped ionacoustic waves of small amplitudes.

Acknowledgements. The authors thank their colleagues from the Department of Space Plasma Physics for continuing support and discussion at seminars. V. Fedun is grateful to STFC (ST/J001430/1) for support received.

Topical Editor M. Gedalin thanks two anonymous referees for their help in evaluating this paper.

\section{References}

Alexandrov, A. F., Bogdankevich, L. S., and Rukhadze, A. A.: Fundamentals of plasma electrodymanics, Vys'shayashkola, Moscow, 1988.

Aschwanden, M.: An evaluation of coronal heating model for active regions based on Yohkoh, SOHO and TRACE observations, Astrophys. J., 560, 1035-1044, 2001.

Duijveman, A., Hoyng, P., and Ionson, I.: Fast plasma heating by anomalous and inertial resistivity effects in the solar atmosphere, Astrophys. J., 245, 721-735, 1981.
Fontenla, J.: Chromospheric plasma and the Farley-Buneman instability in solar magnetic regions, Astron. Astrophys., 442, 1099 1103, 2005.

Fontenla, J., Avrett, E., and Loeser, R.: Energy balance in solar transition region. III. Helium emission in hydrostatic, constantabundance models with diffusion, Astrophys. J., 406, 319-345, 1993.

Fontenla, J., Peterson, W., and Harder, J.: Chromospheric heating by the Farley-Buneman instability, Astron. Astrophys., 480, 839846, 2008.

Foukal, P. and Hinata, S.: Electric fields in the solar atmosphere: a review, Solar Phys., 132, 307-334, 1991.

Galeev, A. and Sagdeev, R.: Nonlinear plasma theory, Problems of Plasma Theory, 7, 3-48, 1973.

Galeev, A. A. and Sagdeev, R. Z.: Current Instabilities and Anomalous Resistivity of Plasma, in: Handbook of Plasma Physics, Basic Plasma Physics, edited by: Galeev, A. A. and Sudan, R. N., North Holland, Amsterdam, Vol. 2, 272-303, 1984.

Galeev, A., Lominadze, D., Pataraia, A., Sagdeev, R., and Stepanov, K.: Anomalous resistance of plasma due to the instability of the cyclotron harmonics, JETP, 417, 1972.

Gogoberidze, G., Voitenko, Y., Poedts, S., and Goossens, M.: Farley-Buneman instability in the Solar chromosphere, Astrophys. J., 706, L12-L16, 2009.

Heyvaerts, J., Priest, E., and Rust, D.: An emerging flux model for the solar flare phenomenon, Astrophys. J., 216, 123-137, 1977.

Holman, G.: Acceleration of runaway electrons and Joule heating in solar flares, Astrophys. J., 293, 584-594, 1985.

Ionson, I.: Resonant absorption of Alfvenic surface waves and the heating of solar coronal loops, Astrophys. J., 226, 650-673, 1978.

Kadomtsev, B.: Collective phenomena in plasma, Nauka, Moscow, 1988 (in Russian).

Kryshtal, A.: Bernstein-wave instability in a collisional plasma with a quasistatic electric field, J. Plasma Phys., 60, 469-484, 1998.

Kryshtal, A. and Gerasimenko, S.: Slow magnetoacoustic-like waves in post-flare loops, Astron. Astrophys., 420, 1107-1115, 2004.

Kryshtal, A. and Kucherenko, V.: Ion-acoustic instability caused by large-scale electric field in solar active regions, Solar Phys., 165, 139-153, 1996.

Kryshtal, A., Gerasimenko, S., and Voitsekhovska, A.: "Oblique" Bernstein modes in solar pre-flare plasma: generation of second harmonics, Adv. Space Res., 5, 791-796, 2012.

Machado, M., Avrett, E., Vernazza, J., and Noyes, R.: Semiempirical models of chromospheric flare regions, Astrophys. J., 242, 336-357, 1980.

McClements, K.: Langmuir wave generation by thick target electron beams in solar flares - The effects of density variations and reverse currents, Astron. Astrophys., 208, 279-286, 1989.

Melnikov, V., Shibasaki, K., and Reznikova, V.: Loop-Top Nonthermal Microwave Source in Extended Solar Flaring Loops, Astrophys. J., 580, L185-L188, 2002.

Miller, I. A., Cargil, P., Emslie A., Holman, G., Dennis, B., La Rosa, T., Wiglee, R., Benka, S., and Tsuneta, S.: Critical issues for understanding particle acceleration in impulsive solar flares, J. Geophys. Res., 102, 14631-14660, 1997.

Pines, D. and Schrieffer, J.: Collective behavior in solid-state plasmas, Phys. Rev., 124, 1387-1400, 1961. 
Solanki, S.: Small-scale solar magnetic fields: an overview, Space Sci. Rev., 63, 1-188, 1993.

Somov, B., Titov, V., and Vemeta, A.: Magnetic reconnection in solar flares, Res. Sci. Technol. Ser. Astromony, 34, 136-237, 1987.

Vernazza, J., Avrett, E., and Loeser, R.: Structure of solar chromosphere. III. Models EUV brightness components of the quiet Sun, Astrophys. J. Suppl. Ser, 45, 635-725, 1981.
Voitenko, Y. and Goossens, M.: Excitation of high-frequency Alfvén waves by plasma outflows from coronal reconnection events, Solar Phys., 206, 285-313, 2002. 